\title{
Painel semântico: revisão sistemática da literatura sobre uma ferramenta imagética de projeto voltada à definição estético- simbólica do produto
}

\section{Moodboards: systematic review of the literature on an imagery design tool focused on the aesthetic-symbolic definition of the product}

\author{
Marcos Roberto dos Reis, Universidade Federal de Santa Catarina.
}

mreisjoi@gmail.com

Eugenio Andrés Díaz Merino, Universidade Federal de Santa Catarina.

eugenio.merino@ufsc.br

\section{Resumo}

O painel semântico é uma ferramenta imagética utilizada no decorrer do processo de definição estética (design) do projeto e tem, por finalidade, servir como referência estético-simbólica da forma e alinhar conceitos semânticos entre os profissionais envolvidos. Apesar de ser utilizado com frequência nos ambientes acadêmico e profissional, pesquisadores pontuam que o painel possui uma quantidade escassa de pesquisas direcionadas a explorar seus procedimentos de construção e aplicações. Este artigo tem como objetivo identificar as pesquisas publicadas que abordam esta ferramenta como principal elemento de estudo. O procedimento de pesquisa envolveu uma revisão sistemática da literatura, com consulta a oito bases de dados. Como resultado, foram identificadas 22 pesquisas, sendo estas divididas em 5 perfis temáticos e analisadas por suas características e contribuições. Observa-se, neste contexto, uma tendência por pesquisas relacionadas à virtualização e automatização do processo de elaboração do painel. Conclui-se que o painel semântico ainda é uma ferramenta com pequena quantidade de pesquisas publicadas e que estas têm sido, em sua maioria, direcionadas a tornar mais colaborativas e ágeis as práticas de seu processo construtivo, contribuindo com a dinâmica dos processos de projeto.

Palavras-chave: Construção do painel semântico, Processo de design, Revisão sistemática.

\begin{abstract}
Moodboard is an imagery tool used during the process of aesthetic definition (design) of the project and is intended to serve as an aesthetic-symbolic reference of the form and to align semantic concepts among the professionals involved. Despite being used frequently in academic and professional environments, researchers point out that the moodboard has a small amount of research aimed at exploring its construction procedures and applications. This article aims to identify published studies that address this tool as a main study element. The research procedure involved a systematic review of the literature, consulting eight databases. As a result, 22 researches were identified, which were divided into 5 thematic profiles and analyzed for their characteristics and contributions. It's possible to notice in this context, that there is a tendency for research related to virtualization and automation of the moodboard elaboration process. It is concluded that the moodboard is still a tool with a small number of published researches and that these have, for the most part, been aimed at making the practices of its construction process more collaborative and rapid, contributing to the dynamics of the design processes.
\end{abstract}

Keywords: Moodboard construction, Design process, Systematic review. 


\section{Introdução}

O processo de design é composto por um conjunto organizado de atividades que possuem, por finalidade, desenvolver a relação entre um produto e seu usuário, tanto em aspectos de interface, quanto estéticos. Dentre as ferramentas que podem ser aplicadas no decorrer deste processo está o painel semântico, sendo apontado como uma importante ferramenta para auxiliar no processo de definição estética no projeto (CASSIDY, 2008; GARNER; MCDONAGH-PHILP, 2001; LUCERO, 2012). É citado também como um instrumento de comunicação entre os envolvidos no projeto de design (BAXTER, 2011; CASSIDY, 2008; DE WET, 2016; ENDRISSAT; ISLAM; NOPPENEY, 2016; GARNER; MCDONAGH-PHILP, 2001) e tem sido ministrado no decorrer de cursos da área de design, utilizado por escritórios de design e departamentos de design de empresas de diversos portes. Classificado como uma ferramenta qualitativa de inspiração visual (CASSIDY, 2011; GARNER; MCDONAGH-PHILP, 2001), sua aplicação no processo de design está em servir como fonte de referência sensorial, especialmente visuais, no emprego de elementos estéticos e simbólicos para a definição da forma de um produto (LÖBACH, 2001). Neste contexto Bruseberg, McDonagh e Wormald (2004, p. 124) colocam que as "imagens são um recurso poderoso para transmitir significados, particularmente valores e experiências emocionais". Para Bürdek (2015, p. 120), seu uso se faz necessário quando "as descrições verbais de objetivos, conceitos e soluções não são mais suficientes, especialmente para o design desenvolvido dentro de um marketing global". Como resultado de sua aplicação, pode-se observar que carros velozes costumam ter uma aparência elegante e aerodinâmica e carros off-road costumam ter aparência rústica e robusta. O painel também é utilizado para alinhar conceitos sobre algum tema específico (LUCERO, 2012), de forma a evitar que interpretações dissonantes, envolvendo semânticas, possam prejudicar consensos entre as pessoas envolvidas. Diminuem-se, assim, as possibilidades de haver diferentes visões sobre um mesmo tema dentro da equipe de projeto.

O painel semântico é uma ferramenta que faz parte da categoria de painéis imagéticos, que possuem como característica principal o uso de referências visuais para orientação da equipe de projetos. Entram nessa categoria: storyboard; painel de estilo de vida; painel de conceitos e o painel semântico (CASSIDY, 2008). As imagens dos painéis podem ser organizadas em diferentes configurações, não possuindo regras específicas. Pereira (2010, p. 39) aponta que

[...] enquanto alguns profissionais optam por separar as referências da prancha em aspectos a serem planejados (paleta de cores, acessórios, ambientes, por exemplo) e outros procuram aproximá-las para facilitar a expressão de ideia, alguns projetistas trabalham com uma lógica de construção aparentemente indefinida.

Os painéis imagéticos (Figura 1) são tecnicamente simples para serem elaborados. Possuem dois elementos básicos: uma base de apoio e uma mensagem a ser transmitida por meio das imagens fixadas a ele. Como base, entenda-se qualquer área, de fácil visualização, reservada para a colocação da mensagem. Pode ser uma placa, uma parede, um tecido, etc. O essencial é que se defina uma área a ser utilizada. Quanto à mensagem, usualmente é composta por um conjunto de imagens selecionadas e apresentadas por meio de impressos fotográficos ou projetores. Podem fazer parte também algumas palavras-chave, frases curtas e, eventualmente, objetos físicos, como folhas ou tecidos. 


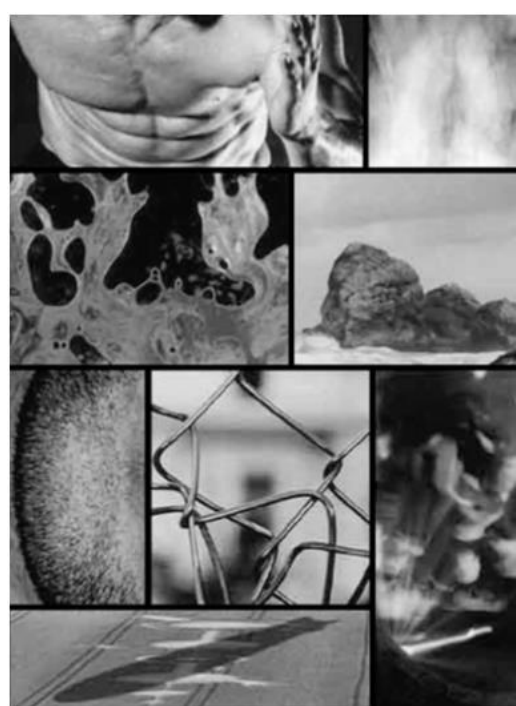

(1)

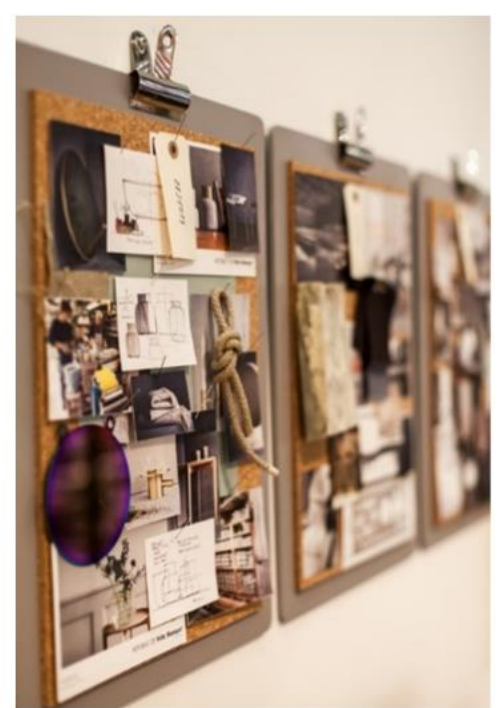

(2)

Figura 1 - Painéis imagéticos com (1) imagens de significados masculinos e (2) a aplicação de imagens e objetos. Fontes: (1)

McDonagh e Denton (2005); (2) Marie Linner (2017).

Considerando que o painel semântico contribui com o alinhamento de conceitos estéticos e fornece referências para a geração de propostas no processo de criação estética, é plausível que se encontrem estudos publicados abordando a ferramenta, suas particularidades e novas possibilidades de uso. Entretanto, Garner e McDonagh-Philp (2001, p. 62) observam que "não existem textos sobre a utilização e a importância dos painéis semânticos na educação de design, na medida em que o ensino do desenho, da ciência dos materiais e da ergonomia são apoiados". Neste sentido, McDonagh e Denton (2005, p. 36) aponta que os painéis "são ensinados há muito tempo a estudantes de arte e design, mas raramente seu uso foi analisado". Este é um entendimento também compartilhado entre outros autores de pesquisas relacionadas ao painel, que citam não encontrar muitas pesquisas com algum aprofundamento nas formas de uso e aplicação da ferramenta (CASSIDY, 2008; FREEMAN; MARCKETTI; KARPOVA, 2017; MCDONAGH; STORER, 2004; PEREIRA, 2010).

Dados os comentários e observações realizados, questiona-se: quais pesquisas foram publicadas sobre as características, formas de uso e aperfeiçoamento, da ferramenta painel semântico? Neste sentido, o objetivo do artigo é apresentar um panorama sobre pesquisas publicadas que tenham o painel semântico como elemento central de estudo. Incluem-se neste escopo, descrições da ferramenta e seu processo de uso, incrementos nos procedimentos e aplicações inovadoras.

\section{Procedimento metodológico}

Esta pesquisa tem, como principais características de classificação, ser de natureza básica, de objetivo descritivo, com abordagem quantitativa, e se utilizar, por procedimento técnico, de pesquisa bibliográfica. Para o recorte temporal, definiu-se que serão considerados os trabalhos publicados entre os anos de 2001 a 2019 e, como critério de exclusão, optou-se por realizar uma pesquisa bibliográfica limitada às publicações em língua inglesa, espanhola e portuguesa, por 
situarem-se dentro do domínio dos autores. Neste sentido, o termo similar ao painel semântico, em língua inglesa, é mood board ou moodboard, sendo encontrado nas duas grafias, e em língua espanhola, é panel semántico.

De maneira a estruturar as publicações encontradas e tornar o processo transparente, replicável e completo, tanto quanto possível, optou-se aqui pela elaboração de uma Revisão Sistemática da Literatura (RSL). Esta caracteriza-se como uma síntese de estudos primários que contém objetivos, materiais e métodos claramente explicitados e que foi conduzida de acordo com uma metodologia clara e reprodutível (GREENHALGH, 1997). Para pesquisar publicações nas bases de dados conhecidas, utilizou-se o protocolo PRISMA ${ }^{1}$ - Preferred Reporting Items for Systematic Reviews and Meta-Analyses - que consiste em um checklist com 27 itens e um fluxograma de quatro etapas.

Inicialmente definiu-se o protocolo de busca (também conhecido por string), que é a composição de palavras e operadores booleanos a serem utilizados nas buscas dentro das bases de dados. Este foi composto pela somatória de palavras relacionados ao painel semântico, um na língua portuguesa, um na língua espanhola e dois na língua inglesa, combinadas pelo operador booleano "OR", apresentando a seguinte estrutura: ("painel semântico" OR "panel semántico" OR "mood board*” OR "moodboard*”).

Para a definição das bases de dados a serem consultadas, utilizou-se como critério de seleção: (1) bases que já são utilizadas pelos autores; e (2) que tenham possibilidade de aplicação filtro de "artigos revisados por pares". Neste sentido, como cada base tem sua seleção de fontes e artigos de acesso direto, é conveniente procurar em mais de um para obter melhor cobertura (NAKANO; MUNIZ JR.; MUNIZ, 2018). No total, foram selecionadas sete bases de dados: ProQuest ${ }^{2}$; Scopus $^{3}$; Periódicos CAPES ${ }^{4}$; JSTOR ${ }^{5}$; Taylor \& Francis Online ${ }^{6}$; Science Direct ${ }^{7}$; Web of Science ${ }^{8}$. Adicionalmente, em um segundo momento, foi incluído o Google Acadêmico ${ }^{9}$ por se tratar de uma base de dados com maior amplitude de informações sobre publicações da internet. Por outro lado, incluem-se nos seus resultados muitos trabalhos da chamada "literatura cinza", que são artigos publicados em revistas não indexadas e sem revisão por pares.

Para o processo de seleção, leitura e análise dos artigos, foram utilizados os softwares Microsoft Excel 2019 e Mendeley Desktop v.1.19.5. O Excel é um conhecido aplicativo de planilha eletrônica e foi utilizado para organizar o processo de análise de duplicidade de artigos entre as bases de dados pesquisadas. O Mendeley, é um aplicativo gratuito de gerenciamento, compartilhamento e normalização de referências bibliográficas, e foi utilizado para organizar o processo de leitura e análise dos artigos selecionados no decorrer das etapas seguintes do PRISMA.

\footnotetext{
${ }^{1}$ Principais Itens para Relatar Revisões Sistemáticas e Meta-Análises, em tradução própria.

${ }^{2}$ https://www.proquest.com/LATAM-PT/.

${ }^{3}$ https://www.scopus.com/search/form.uri?display=basic.

${ }^{4}$ http://www-periodicos-capes-gov-br.ez372.periodicos.capes.gov.br.

5 https://www.jstor.org.

6 https://tandfonline.com.

7 https://www.sciencedirect.com.

8 https://clarivate.com/products/web-of-science/.

9 https://scholar.google.com.br.
} 
Após a aplicação do protocolo PRISMA, para a busca e seleção de artigos, foi realizada a classificação das pesquisas, que compreende a identificação das principais características de pesquisa de cada artigo segundo os seguintes pontos de vista (GIL, 2017; SILVA; MENEZES, 2005):

- Natureza: básica ou aplicada;

- Abordagem: qualitativa, quantitativa ou quali-quanti;

- Objetivos: exploratória, descritiva ou explicativa;

- Procedimentos: pesquisa bibliográfica, pesquisa documental, pesquisa experimental, levantamento, estudo de caso, pesquisa-ação ou pesquisa participante.

Em seguida, os artigos foram divididos em cinco diferentes temas: melhorias na indexação de imagens; interatividade e realidade virtual; co-design; leitura do painel; e, caracterizar e discutir. Os temas foram criados com base nas palavras-chave e objetivos de cada artigo, de modo a facilitar a organização para consultas. Por último, foram dispostos por ordem temporal com o objetivo de destacar a quantidade de artigos publicados em cada ano e as linhas temáticas desenvolvidas.

\section{Desenvolvimento da RSL}

A presente RSL foi desenvolvida inicialmente em novembro de 2018 e atualizada em setembro de 2019 (Figura 2). Para a primeira etapa - Identificação - que se refere à coleta de resultados a partir da aplicação da string de busca em bases de dados bibliográficos, utilizou-se das bases de dados que possibilitavam o uso do filtro "revisão por pares".

Após a consulta inicial, utilizando-se o protocolo de busca e a opção "revisão por pares" ativa, o resultado obtido foi de 576 artigos encontrados. Suas informações principais (título, autor, ano de publicação e periódico) foram salvas e colocados na planilha Excel para proceder uma verificação de duplicidade. O procedimento apontou 146 casos, reduzindo o total para 430 artigos. Neste momento, foi realizada uma consulta paralela ao Google Acadêmico para verificar se haveria algum resultado que não tivesse sido abrangido pelas demais bases, tendo como resultado 4030 artigos encontrados. Optou-se aqui por manter o mesmo protocolo de busca utilizado para as sete bases consultadas anteriormente.

Na segunda etapa - Triagem - foi feita uma seleção a partir da leitura de título, resumo e palavras chave de cada um dos 430 artigos encontrados nas bases de dados bibliográficos. Destes, foram selecionados 37 artigos para a etapa seguinte. Estes artigos foram também utilizados como referência para a triagem dos resultados encontrados pelo Google Acadêmico. Neste caso, procedeu-se à leitura de títulos, palavras-chave e, quando necessário, resumos. Desta maneira, reduziu-se a quantidade de resultados selecionados no Google Acadêmico para 10. No total, 47 artigos foram selecionados para leitura completa.

$\mathrm{Na}$ terceira etapa - Elegibilidade - foram definidos alguns critérios de inclusão para selecionar os artigos que apresentam uma contribuição relevante sobre ou para a ferramenta:

1) Análises sobre a ferramenta painel semântico ou alguma de suas características - 7 artigos;

2) Aperfeiçoamento no uso da ferramenta painel semântico - 13 artigos;

3) Aplicação específica (beneficiada direta e/ou essencialmente pelo uso da ferramenta painel semântico) - 2 artigos. 
Ao final, foram selecionados 22 artigos publicados e que contribuem com alguma melhoria, aplicação ou discussão sobre a ferramenta painel semântico.

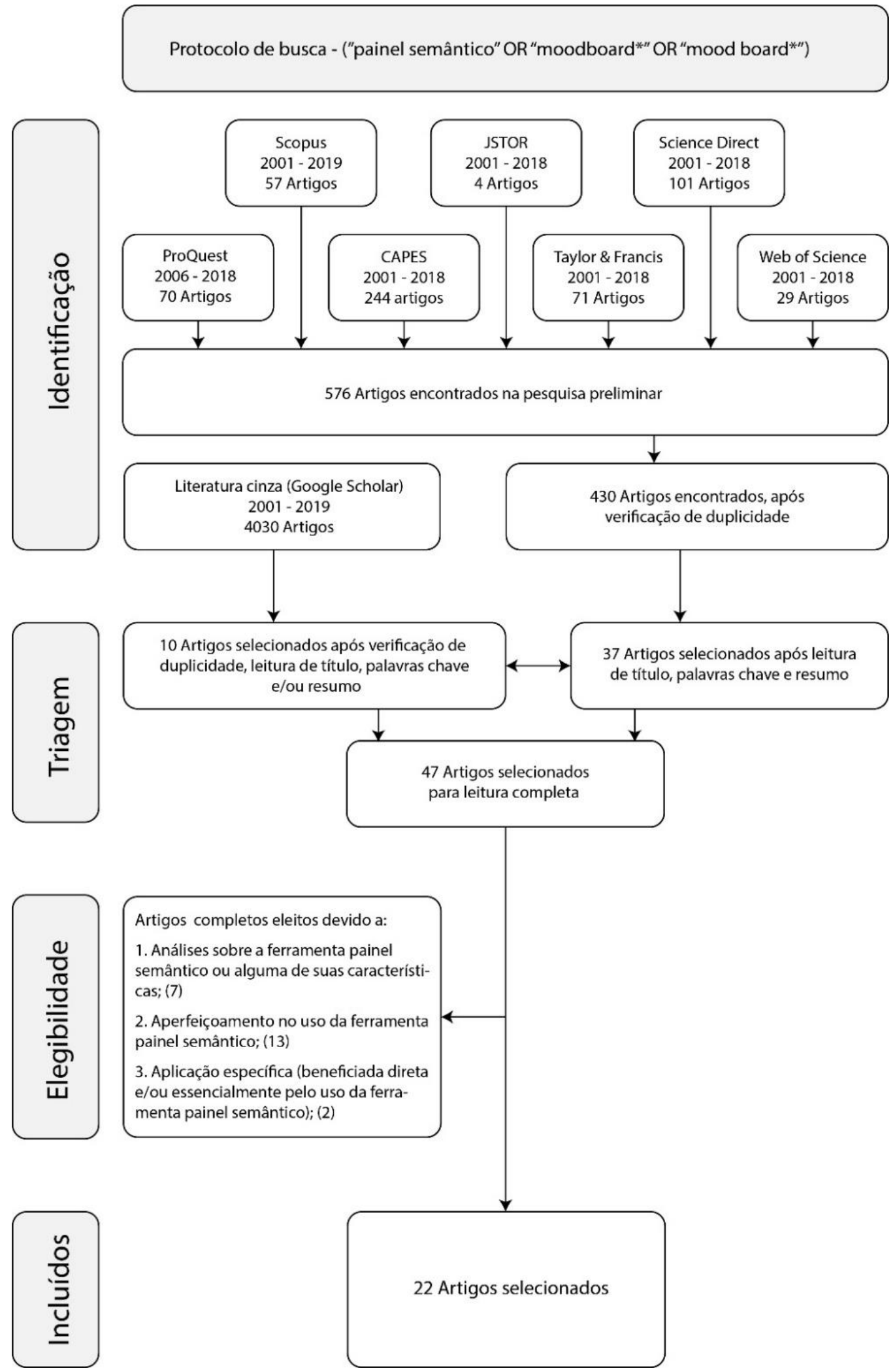

Figura 2 - Aplicação do protocolo PRISMA na RSL. Fonte: elaborado pelo autor. 


\section{Classificação das pesquisas}

Os 22 artigos foram classificados por suas características de pesquisa (Quadro 1). Em relação à natureza, existe um amplo predomínio da pesquisa aplicada, com 95,45\% (21 artigos) dos casos, tendo a pesquisa básica apenas 4,55\% (1 artigo). Quanto ao tipo de abordagem, a aplicação de métodos qualitativos, de forma exclusiva, aparece em 50\% (11 artigos) das pesquisas, já as exclusivamente quantitativas representam 27,27\% (6 artigos), e as mistas (quali-quanti) estão em $22,73 \%$ (5 artigos) delas. Os objetivos são exploratórios em 54,55\% (12 artigos) das pesquisas e explicativos nos 45,45\% (10 artigos) restantes. Não houve ocorrência de pesquisa descritiva. Em relação aos procedimentos, existe um predomínio da pesquisa experimental, com 54,55\% (12 artigos), seguido por pesquisa-ação com 27,27\% (6 artigos) e estudo de caso com 18,18\% (4 artigos). Não houve ocorrência de trabalhos que utilizassem procedimentos de pesquisa bibliográfica, documental, participante ou de levantamento. Observe-se que todas as pesquisas com objetivos do tipo explicativo utilizaram, como procedimento, o tipo experimental, mostrando uma tendência por esta associação ao buscar registrar e analisar os fenômenos estudados, além de identificar suas causas (SEVERINO, 2013). Por outro lado, todos os procedimentos do tipo estudo de caso e pesquisa-ação aparecem associados a um objetivo de caráter exploratório.

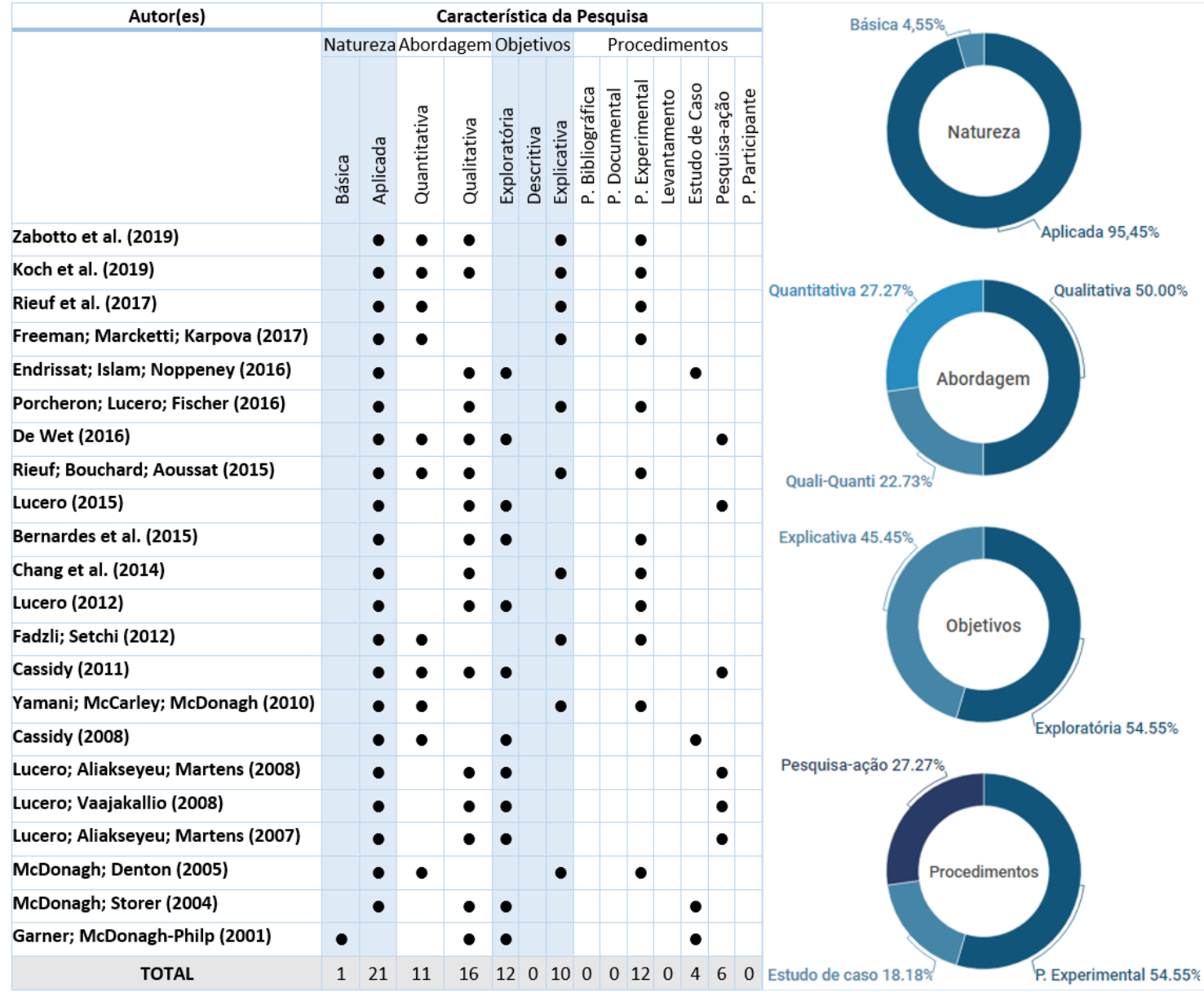

Quadro 1 -Característica de pesquisa dos artigos. Fonte: elaborado pelo autor. 


\section{Resultados e discussão}

Nesta seção são apresentados os resultados e discussões relacionados aos artigos encontrados através da RSL. As temáticas, com seus artigos relacionados, possuem as seguintes características, composição e descrição:

- Melhorias na Indexação de Imagens: abrange cinco artigos que tem por finalidade propor melhorias no processo de seleção de imagens por meio de opiniões das partes envolvidas (especialistas ou clientes) utilizando ferramentas de apoio.

- De Wet (2016) - propõe usar uma sequência de cinco ações, sobre imagens escolhidas, para melhoria na qualidade delas;

- Bernardes et al. (2015) - propõem usar a opinião de especialistas (e clientes) para catalogar as imagens via engenharia Kansei, com vistas ao desenvolvimento de um software;

- Fadzli; Setchi (2012) - propõem usar um algoritmo chamado SDNA (Semantic DNA) para classificar imagens para o painel.

- Interatividade e Realidade Virtual: a presença e facilidades que computadores, projetores, internet e telefones celulares tem oferecido, aliados a uma integração cada vez mais eficiente entre eles, criaram um ambiente propício para esta linha de pesquisa.

- Zabotto et al. (2019) - investigam a possibilidade de usar um algoritmo, por meio da engenharia Kansei, para automatizar o processo de classificação de imagens voltadas ao painel semântico;

- Koch et al. (2019) - apresentam um sistema IA interativo, cooperativo e contextualizado para contribuir no processo de seleção de imagens para o painel semântico;

- Rieuf et al. (2017) - fazem uma análise comparativa entre construção virtual e tradicional do painel semântico;

- Porcheron; Lucero; Fischer (2016) - propõem usar um aplicativo mobile para organizar o processo de criação do painel;

- Rieuf; Bouchard; Aoussat (2015) - comparam um ambiente tradicional com outro imersivo (virtual) na leitura de painel semântico e sua identificação com o Kansei;

- Lucero; Aliakseyeu; Martens (2008) - apresentam uma parede interativa (Funky Wall) para exibição do painel semântico;

- Lucero; Aliakseyeu; Martens (2007) - estudam o uso de mesa interativa para construir o painel semântico.

- Co-design: tem como linha a construção de espaços interativos, para diálogo e interação, voltados ao processo de construção de painéis semânticos. Para tanto, se utilizam de sistemas conectados (computador e/ou celular) em um espaço físico adaptado.

○ Lucero (2015) - estuda a construção de painel para conceber o espaço interativo Funky Design Spaces (co-design);

- Lucero; Vaajakallio (2008) - propõem um espaço de diálogo (co-design) voltado para construção do painel semântico.

- Leitura do Painel: os processos de leitura são fundamentados em percepções subjetivas dos envolvidos. Neste sentido os artigos apresentam pesquisas que se utilizam destas percepções para outras finalidades que não apenas as relacionadas à estética de um projeto.

- Freeman; Marcketti; Karpova (2017) - propõem uma ferramenta para avaliar a criatividade das imagens utilizadas nos painéis semânticos criados;

○ Endrissat; Islam; Noppeney (2016) - utilizam o painel semântico para alinhar conceitos durante um projeto;

- Chang et al. (2014) - avaliam qualidades emocionais das pessoas através da interpretação de painéis semânticos;

- Yamani; Mccarley; Mcdonagh (2010) - comparam a diferença de leitura (julgamento) entre jovens e idosos; 
- Mcdonagh; Denton (2005) - exploram a percepção compartilhada (de imagens do painel semântico) por parte de alunos de design.

- Caracterizar e Discutir: abrange artigos que abordam o painel semântico como foco central de estudo, tratando de explorar características da ferramenta, porém de maneira ampla, sem se ater a algum aspecto específico.

- Lucero (2012) - propõem associar o painel semântico a cinco benefícios (enquadramento, alinhamento, paradoxo, abstração e direção);

- Cassidy (2011) - explora o processo de construção do painel semântico;

- Cassidy (2008) - apresenta um panorama geral sobre as práticas correntes do processo de ensino e aprendizagem do painel semântico;

- Mcdonagh; Storer (2004) - apresentam uma exploração sobre os potenciais usos do painel semântico como ferramenta de design e suporte de projeto;

- Garner; Mcdonagh-Philp (2001) - definem e discutem o que é o painel semântico no contexto da educação em design.

Em relação ao idioma, a quase totalidade dos artigos selecionados (90,9\% - 20 artigos) está em inglês, dois artigos estão em português e nenhum em espanhol. Os artigos em inglês estão presentes em todas as áreas temáticas. Em português aparecem em duas - Melhorias na Indexação de Imagens e Interatividade e Realidade Virtual - e tem, em comum, direcionamentos voltados à construção de software para auxiliar na seleção de imagens para o painel.

Na Figura 3 os artigos estão organizados em ordem temporal, estando também divididos por sua temática e perfil de publicação (periódicos na cor vermelha e congressos na cor azul).

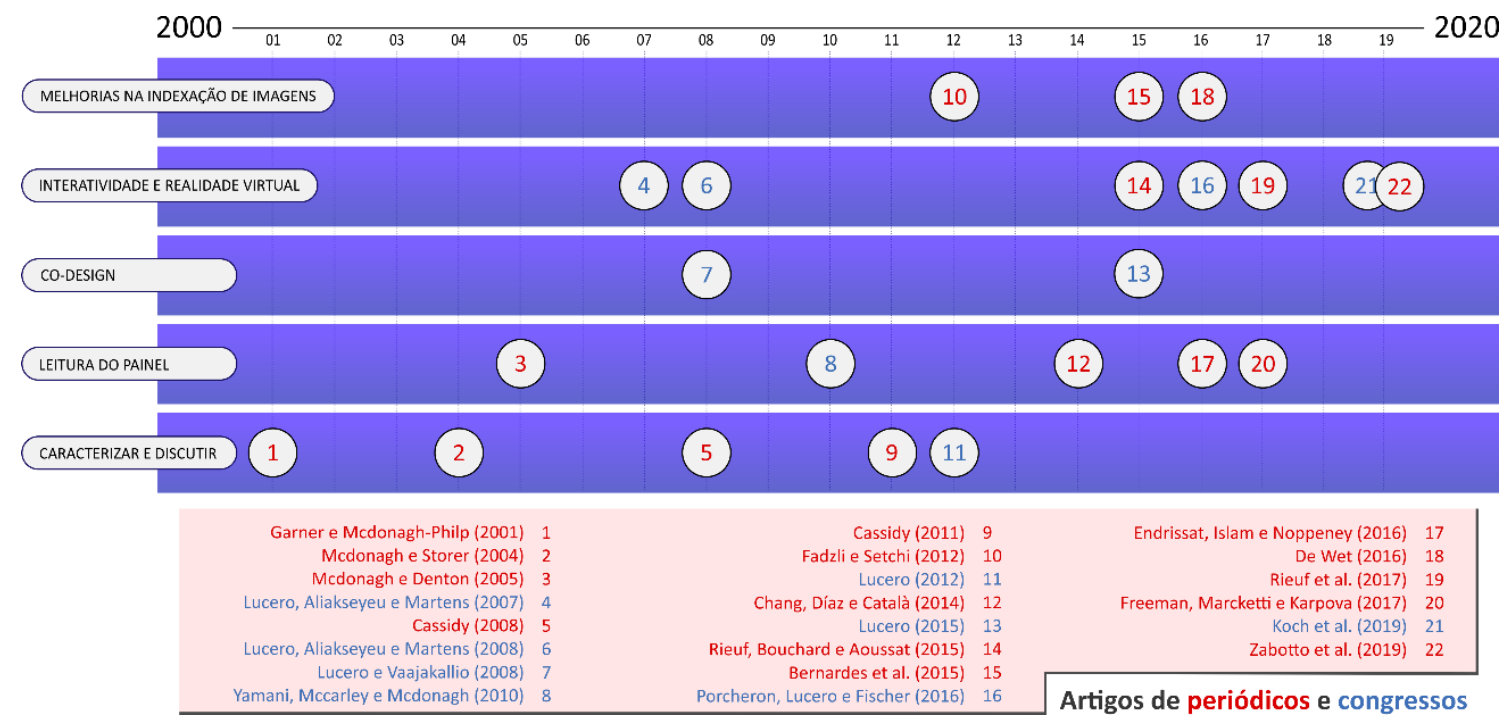

Figura 3 - Cronologia dos artigos. Fonte: elaborado pelo autor.

Observa-se que o tema Interatividade e Uso de Realidade Virtual tem sido objeto de maior atenção nas pesquisas publicadas mais recentemente, tendo cinco artigos listados nos últimos quatro anos. Coincide com a massificação dos smartphones e aplicativos que, entre outros benefícios, colocou literalmente na palma da mão muitas soluções relacionadas à interação e aplicações em realidade virtual e realidade aumentada. Esta tendência também é observada no tema Melhorias na Indexação de Imagens, que possui um viés na linha da interatividade, com duas pesquisas recentes voltadas ao aprimoramento de justificativas técnicas para as imagens inseridas no painel. O tema Leitura do Painel, tem sido abordado com mais frequência nos últimos cinco anos e direcionadas à identificação de características psicológicas das pessoas. São derivações de uso do painel e procuram oferecer alguma objetividade ao aspecto subjetivo da 
leitura. O tema Caracterizar e Discutir teve artigos publicados até o ano de 2012. Desde então, não tem sido mais objeto de novos trabalhos, o que pode indicar desinteresse em estudar com maior profundidade as características objetivas e subjetivas que a ferramenta possui.

As pesquisas apontam também para uma tendência de virtualização do processo, utilizandose de: tecnologia para capturar movimentos; internet e celulares para compartilhar imagens; projetores e realidade virtual para expor e aprimorar os resultados; e algoritmos para direcionar as escolhas por imagens com melhor aderência ao tema pesquisado. Os resultados são apresentados como promissores, mas ainda se encontram no campo dos experimentos e comparação de resultados com procedimentos em painéis físicos. Pode-se inferir que irão suplantar estes, em grande parte e num futuro próximo, por meio de suas vantagens, como rapidez, fluidez, conectividade, apoio de inteligência artificial e colaboração entre usuários.

Observam-se também pesquisas que investigam usos do painel dentro de contextos relacionado à criatividade e qualidades emocionais, sugerindo novas aplicações e propondo que a interpretação das imagens colocadas nele possam ser utilizadas para identificar características emocionais sobre a pessoa que desempenhou a atividade. São aplicações que fogem do contexto mais difundido, direcionando-se a outras possibilidades de emprego para a composição de imagens do painel, aproximando-se da área da psicologia.

Constatou-se ainda pesquisas com objetivos de criar técnicas para auxiliar na melhoria do processo de escolha de imagens, evitando o excesso de subjetivismo nesta tarefa, e direcionando ao uso de critérios mais objetivos para a execução desta tarefa.

\section{Conclusão}

De acordo com os artigos apresentados, o painel semântico é percebido como uma ferramenta de referência estético-simbólica e como um espaço adequado para produzir alinhamento conceitual e inspiração sensorial para designers. É visto como um elemento importante no processo de definição estética (design) dos projetos e que poderia ser melhor explorado em diferentes formas e aplicações. Ter ciência sobre as pesquisas relacionadas ao painel auxilia a compreender melhor as possibilidades que a ferramenta oferece, aplicações em que pode ser útil, e melhorias que estão sendo estudadas. Neste sentido, buscou-se resposta para a seguinte questão de pesquisa: quais pesquisas foram publicadas sobre as características, formas de uso e aperfeiçoamento, da ferramenta painel semântico? Para tanto, realizou-se a RSL e procedeu-se com a análise dos dados de 22 artigos selecionados. Entre os resultados estão uma tabela-síntese de todos os artigos, possibilitando acesso facilitado às principais informações e resultados de cada um, uma análise das características de pesquisa e as principais áreas temáticas abordadas. Possibilita-se assim, uma visão abrangente sobre pesquisas direcionadas à ferramenta, servindo como ponto de partida para novos estudos voltados ao painel semântico, especificamente, ou aos painéis imagéticos, em um foco mais expandido.

Persiste, contudo, a percepção de que é uma ferramenta pouco investigada por pesquisadores. Pôde-se perceber, por meio da realização da RSL, a pequena quantidade de trabalhos identificados que possuem o painel semântico como elemento central da pesquisa. Destaque-se aqui que, na elaboração do protocolo de busca, não houve necessidade de acrescentar outros termos, além da 
sua própria denominação em português, espanhol e inglês, para filtrar os resultados encontrados, reforçando esta percepção.

Este trabalho procura colaborar com a organização e divulgação das pesquisas relacionadas a esta ferramenta, e espera incentivar o desenvolvimento de outras, que contribuam com inovações em seu processo, aplicações e/ou resultados. Como sugestão para futuras pesquisas, o painel semântico pode ser explorado com maior profundidade em suas características construtivas, estudando meios ou procedimentos que produzam painéis mais bem alinhados com a visão de determinado público alvo, podendo envolve-lo de maneira mais significativa. Neste sentido sugere-se a exploração de aspectos relacionados à protocolos de tarefas, como busca, seleção e posicionamento de imagens.

\section{Referências}

BAXTER, M. Projeto de Produto: guia prático para o design de novos produtos. Traducao Itiro Iida. 3. ed. São Paulo: Edgard Blücher, 2011.

BERNARDES, A. M. et al. Kanseiboards : Proposta de aplicação de Engenharia Kansei para a catalogação de referências criativas e construção de mood boards em mídia digital. Educação Gráfica (UNESP Bauru), v. 19, n. 1, p. 44-62, 2015.

BRUSEBERG, A.; MCDONAGH, D.; WORMALD, P. The use of images to elicit user needs for the design of playground equipment. In: MCDONAGH, D. et al. (Eds.). . Design and Emotion. London: CRC Press, 2003. p. 114-118.

BÜRDEK, B. E. Design: History, Theory and Practice of Product Design. 2. ed. Basel: Birkhäuser Basel, 2015.

CASSIDY, T. The Mood Board Process Modeled and Understood as a Qualitative Design Research Tool. Fashion Practice, v. 3, n. 2, p. 225-251, 27 nov. 2011.

CASSIDY, T. D. Mood boards: Current practice in learning and teaching strategies and students' understanding of the process. International Journal of Fashion Design, Technology and Education, v. 1, n. 1, p. 43-54, mar. 2008.

CHANG, H.-M. et al. Mood Boards as a Universal Tool for Investigating Emotional Experience. In: Lecture Notes in Computer Science (including subseries Lecture Notes in Artificial Intelligence and Lecture Notes in Bioinformatics). [s.l: s.n.]. v. 8520 LNCSp. 220-231.

DE WET, A. J. C. C. An educational tool to encourage higher level thinking skills in the selection of images for fashion design mood boards: an action research approach. International Journal of Fashion Design, Technology and Education, v. 10, n. 1, p. 1625, 2 jan. 2016.

ENDRISSAT, N.; ISLAM, G.; NOPPENEY, C. Visual organizing: Balancing coordination and creative freedom via mood boards. Journal of Business Research, v. 69, n. 7, p. 23532362, jul. 2016.

FADZLI, S. A.; SETCHI, R. Concept-based indexing of annotated images using semantic DNA. Engineering Applications of Artificial Intelligence, v. 25, n. 8, p. 1644-1655, dez. 2012.

FREEMAN, C.; MARCKETTI, S.; KARPOVA, E. Creativity of images: using digital consensual assessment to evaluate mood boards. Fashion and Textiles, v. 4, n. 1, p. 17, 28 dez. 2017.

GARNER, S.; MCDONAGH-PHILP, D. Problem Interpretation and Resolution via Visual 
Stimuli: The Use of "Mood Boards" in Design Education. International Journal of Art and Design Education Design Education, v. 20, n. 1, p. 57-64, fev. 2001.

GIL, A. C. Como elaborar projetos de pesquisa. 5. ed. São Paulo: Atlas, 2017.

GREENHALGH, T. Papers that summarize other papers (systematic review and metaanalyses). British Medical Journal, v. 315, n. 7109, p. 672-675, 1997.

KOCH, J. et al. May AI? Proceedings of the 2019 CHI Conference on Human Factors in Computing Systems - CHI '19. Anais...New York, New York, USA: ACM Press, 2019Disponível em: <http://dl.acm.org/citation.cfm?doid=3290605.3300863>

LÖBACH, B. Design Industrial: bases para a configuração dos produtos industriais. São Paulo: Edgard Blücher, 2001.

LUCERO, A. Framing, aligning, paradoxing, abstracting, and directing: How design mood boards work. Designing Interactive Systems Conference, DIS '12. Anais...New York: ACM Press, 2012Disponível em: <http://www.scopus.com/inward/record.url?eid=2-s2.084864745104\&partnerID=40\&md5=9f0b7d16c7c1857bd578e4b5d1c22432>

LUCERO, A. Funky-Design-Spaces: Interactive Environments for Creativity Inspired by Observing Designers Making Mood Boards. In: ABASCAL, J. et al. (Eds.). . Lecture Notes in Computer Science (including subseries Lecture Notes in Artificial Intelligence and Lecture Notes in Bioinformatics). Lecture Notes in Computer Science. Cham: Springer International Publishing, 2015. v. 9298p. 474-492.

LUCERO, A.; ALIAKSEYEU, D.; MARTENS, J.-B. Augmenting Mood Boards: Flexible and Intuitive Interaction in the Context of the Design Studio. Second Annual IEEE International Workshop on Horizontal Interactive Human-Computer Systems (TABLETOP'07). Anais...IEEE, out. 2007Disponível em: <http://ieeexplore.ieee.org/document/4384100/>

LUCERO, A.; ALIAKSEYEU, D.; MARTENS, J.-B. Funky wall: Presenting mood boards using gesture, speech and visuals. Proceedings of The Working Conference on Advanced Visual Interfaces -- AVI '08. Anais...New York, New York, USA: ACM Press, 2008Disponível em: <http://portal.acm.org/citation.cfm?doid=1385569.1385650>

LUCERO, A.; VAAJAKALLIO, K. Co-Designing Mood Boards: Creating Dialogue with People. Proc. of IASTED HCI 2008, p. 254-260, 2008.

MARIE LINNER. Mood boards on a white painted wall. Disponível em: <https://image.shutterstock.com/image-photo/stockholm-february-2017-mood-boards-450w747094129.jpg>. Acesso em: 14 jan. 2019.

MCDONAGH, D.; DENTON, H. Exploring the degree to which individual students share a common perception of specific mood boards: observations relating to teaching, learning and team-based design. Design Studies, v. 26, n. 1, p. 35-53, jan. 2005.

MCDONAGH, D.; STORER, I. Mood Boards as a Design Catalyst and Resource: Researching an Under-Researched Area. The Design Journal, v. 7, n. 3, p. 16-31, 28 nov. 2004.

NAKANO, D.; MUNIZ JR., J.; MUNIZ, J. Writing the literature review for empirical papers. Production, v. 28, n. 0, 2018.

PEREIRA, T. V. Mood Board como espaço de construção de metáforas. [s.l.] UNISINOS, 2010.

PORCHERON, M.; LUCERO, A.; FISCHER, J. E. Co-Curator: Designing for Mobile Ideation in Groups. Proceedings of the 20th International Academic Mindtrek Conference on - AcademicMindtrek '16. Anais...New York, New York, USA: ACM Press, 2016Disponível em: <http://dl.acm.org/citation.cfm?doid=2994310.2994350>

RIEUF, V. et al. Emotional activity in early immersive design: Sketches and moodboards in 
virtual reality. Design Studies, v. 48, p. 43-75, jan. 2017.

RIEUF, V.; BOUCHARD, C.; AOUSSAT, A. Immersive moodboards, a comparative study of industrial design inspiration material. J. of Design Research, v. 13, n. 1, p. 78-106, 2015.

SEVERINO, A. J. Metodologia do trabalho científico. 23. ed. São Paulo: Cortez, 2013.

SILVA, E. L. DA; MENEZES, E. M. Metodologia da Pesquisa e Elaboração de Dissertação. 4. ed. Florianópolis: UFSC, 2005.

YAMANI, Y.; MCCARLEY, J. S.; MCDONAGH, D. Transgenerational communication through affective imagery in mood boards. Proceedings of the Human Factors and Ergonomics Society Annual Meeting, v. 54, n. 20, p. 1762-1765, set. 2010.

ZABOTTO, C. N. et al. Automatic digital mood boards to connect users and designers with kansei engineering. International Journal of Industrial Ergonomics, v. 74, n. July, p. 11, 2019.

\section{Sobre os autores}

Marcos Roberto dos Reis. Possui graduação em Desenho Industrial pela Universidade Federal do Paraná, mestrado em Engenharia Mecânica pela Universidade Federal de Santa Catarina e é doutorando em Engenharia de Produção pela Universidade Federal de Santa Catarina. Atualmente é professor adjunto da Universidade Federal da Fronteira Sul.

mreisjoi@gmail.com

Eugenio Andrés Díaz Merino. Possui graduação em Desenho Industrial pela Universidade Federal do Rio de Janeiro, mestrado em Engenharia de Produção pela Universidade Federal de Santa Catarina e doutorado em Engenharia de Produção pela Universidade Federal de Santa Catarina. Atualmente é professor Titular da Universidade Federal de Santa Catarina e coordena o Núcleo de Gestão de Design e o Laboratório de Design e Usabilidade.

eugenio.merino@ufsc.br 